\title{
Towards Fault-Tolerant Digital Microfluidic Lab-on-Chip: Defects, Fault Modeling, Testing, and Reconfiguration
}

\author{
Krishnendu Chakrabarty \\ Department of Electrical and Computer Engineering \\ Duke University, Durham, NC 27708, USA
}

\begin{abstract}
Dependability is an important attribute for microfluidic lab-on-chip devices that are being developed for safety-critical applications such as point-of-care health assessment, air-quality monitoring, and food-safety testing. Therefore, these devices must be adequately tested after manufacture and during bioassay operations. This paper presents a survey of early work on fault tolerance in digital microfluidic lab-on-chip systems. Defects are related to logical fault models that can be viewed not only in terms of traditional shorts and opens, but which also target biochip functionality. Based on these fault models, test techniques for lab-on-chip devices and digital microfluidic modules are presented.
\end{abstract}

\section{Introduction}

Recent advances in microfluidics technology have led to the emergence of miniaturized lab-on-chip devices for biochemical analysis [Fair 2003, Verpoorte 2003, Dittrich 2006]. Lab-on-chip devices offer the advantages of higher sensitivity, lower cost due to smaller sample and reagent volumes, higher levels of system integration, and less likelihood of human error.

Many commercially-available lab-on-chip are based on continuous fluid flow in permanently-etched microchannels [Verpoorte 2003]. An alternative category of microfluidics, referred to as "digital microfluidics", relies on the principle of electrowetting-on-dielectric [Fair 2003, Cho 2003]. Bioassay protocols are scaled down (in terms of liquid volumes and assay times), and run on a microfluidic chip by manipulating discrete droplets of nanoliter volume using a patterned array of electrodes. By reducing sample and reagent consumption, digital microfluidic lab-on-chip enable continuous sampling and analysis for on-line, real-time, chemical and biological analysis. These systems also have dynamic reconfigurability, whereby microfluidic modules can be relocated to other places on the electrode array, without affecting the functionality, during the concurrent execution of a set of bioassays. Reconfigurability enables the design of multifunctional and "smart" microfluidic lab-on-chip that can be used for a wide variety of applications. Moreover, defects can be tolerated through system reconfiguration after testing and fault diagnosis.

Recent years have seen rapid progress in the mapping of different bioassays for concurrent execution on a digital microfluidic platform. As a result, system complexity and integration levels are increasing steadily. As in the case of integrated circuits, an increase in the density and area of microfluidics-based biochips will lead to higher defect rates thereby reducing yield, especially for newer technologies. However, dependability is an important system attribute for biochips. Dependability is essential for safety-critical applications such as point-of care diagnostics, health assessment and screening for infectious diseases, air-quality monitoring, and food-safety tests, as well as for pharmacological procedures for drug design and discovery that require high precision levels. Some manufacturing defects may be latent, and they may produce errors during field operation. In addition, harsh operational environments and biological samples (e.g., proteins) may introduce physical defects such as particle contamination and residue on surfaces due to adsorption.

Since fluidic operations are repeatedly executed with high precision in compact microfluidic arrays, a group of cells is repeatedly required to perform a large number of operations. Structural test methods, which use test droplets to traverse the target array, do not provide a sufficient level of confidence that these fluidic operations can be reliably performed on the array [Xu 2007a]. For instance, some unit cells, i.e., electrodes, may function correctly during droplet transportation, but they might malfunction during droplet dispensing from reservoirs. Likewise, unit cells that can be reliably combined to operate as a mixer may malfunction when they are used for droplet splitting. Therefore, it is important to carry out functional testing to verify the integrity of the underlying microfluidic platform. To ensure that manufactured biochips are competitive in the emerging low-cost market for disposable biochips and to avoid electrode degradation resulting from excessive actuation, test methodologies should be inexpensive, quick, and effective.

This paper presents a survey of research on fault tolerance in digital microfluidic lab-on-chip. Defects are related to logical fault models that can be viewed not only in terms of traditional shorts and opens, but which also target biochip functionality. Based on these fault models, test techniques for lab-on-chip devices and digital microfluidic modules are presented.

\section{Overview of Digital Microfluidics}

A digital microfluidic biochip utilizes the phenomenon of electrowetting to manipulate and move nanoliter droplets containing biological samples on a two-dimensional electrode array [Fair 2003]. A unit cell in the array includes a pair of electrodes that acts as two parallel plates. The bottom plate contains a patterned array of individually controlled electrodes, and the top plate is coated with a continuous ground electrode. A droplet rests on a hydrophobic surface over an electrode, as shown in Fig. 1. It is moved by applying a control voltage to an electrode adjacent to the droplet and, at the same time, deactivating the electrode just under the droplet. This electronic method of wettability control creates interfacial tension gradients that move the droplets to the charged electrode. Using the electrowetting phenomenon, droplets can be moved to any location on a two-dimensional array.

By varying the patterns of control-voltage activation, many fluid-handling operations such as droplet merging, splitting, mixing, and dispensing can be executed in a similar manner. For example, mixing can be performed by routing two droplets to the same location and then turning them about some pivot points. The digital microfluidic platform offers the additional advantage of flexibility, referred to as reconfigurability, since fluidic operations can be performed anywhere on the array. Droplet routes and 
operation scheduling result are programmed into a microcontroller that drives electrodes in the array. In addition to electrodes, optical detectors such as LEDs and photodiodes are also integrated in digital microfluidic arrays to monitor colorimetric bioassays [Fair 2003].

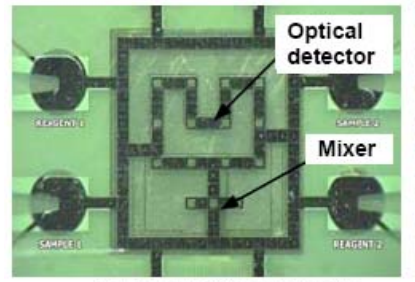

Glass-substrate platform

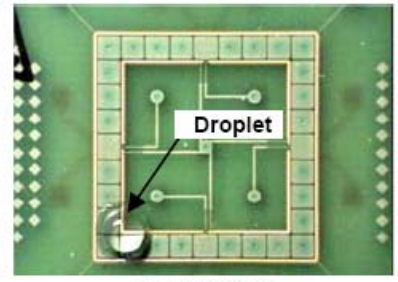

PCB platform
Fig. 1: Fabricated digital microfluidic arrays (Figures courtesy: www.ee.duke.edu/research/microfludics and www.liquid-logic.com).

\section{Fault Modeling}

Like microelectronic circuits, a defective microfluidic biochip is said to have a failure if its operation does not match its specified behavior [Kerkhoff 2007]. In order to facilitate the detection of defects, fault models that efficiently represent the effect of physical defects at some level of abstraction are required. These models can be used to capture the effect of physical defects that produce incorrect behaviors in the electrical or fluidic domain. As described in [Su 2003], faults in digital microfluidic systems can be classified as being either catastrophic or parametric. Catastrophic faults lead to a complete malfunction of the system, while parametric faults cause degradation in the system performance. A parametric fault is detectable only if this deviation exceeds the tolerance in system performance.

Catastrophic may be caused by a number of physical defects, for example:

- Dielectric breakdown: The breakdown of the dielectric at high voltage levels creates a short between the droplet and the electrode. When this happens, the droplet undergoes electrolysis, thereby preventing further transportation.

- Short between the adjacent electrodes: If a short occurs between two adjacent electrodes, the two electrodes effectively form one longer electrode. When a droplet resides on this electrode, it is no longer large enough to overlap the gap between adjacent electrodes. As a result, the actuation of the droplet can no longer be achieved.

- Degradation of the insulator: This degradation effect is unpredictable and may become apparent gradually during the operation of the microfluidic system. A consequence is that droplets often fragment and their motion is prevented because of the unwanted variation of surface tension forces along their flow path.

- Open in the metal connection between the electrode and the control source: This defect results in a failure in activating the electrode for transport.

Table 1 lists some common failure sources, defects and the corresponding fault models for catastrophic faults in digital microfluidic lab-on-chip. Examples of some common parametric faults include the following:

- Geometrical parameter deviation: The deviation in insulator thickness, electrode length and height between parallel plates may exceed their tolerance value.

- Change in viscosity of droplet and filler medium. These can occur during operation due to an unexpected biochemical reaction, or changes in operational environment, e.g., temperature variation.

\section{Structural Test Techniques}

A unified test methodology for digital microfluidic biochips has recently been presented, whereby faults can be detected by controlling and tracking droplet motion electrically [Su 2005a]. Test stimuli droplets containing a conductive fluid (e.g., KCL solution) are dispensed from the droplet source. These droplets are guided through the unit cells following the test plan towards the droplet sink, which is connected to an integrated capacitive detection circuit. Most catastrophic faults result in a complete cessation of droplet transportation. Therefore, we can determine the fault-free or faulty status of the system by simply observing the arrival of test stimuli droplets at selected ports. An efficient test plan ensures that testing does not conflict with the normal bioassay, and it guides test stimuli droplets to cover all the unit cells available for testing. The microfluidic array can be modeled as an undirected graph, and the pathway for the test droplet can be determined by solving the Hamiltonian path problem [Su 2006]. With negligible hardware overhead, this method also offers an opportunity to implement self-test for microfluidic systems and therefore eliminate the need for costly, bulky, and expensive external test equipment. Furthermore, after detection, droplet flow paths for bioassays can be reconfigured dynamically such that faulty unit cells are bypassed without interrupting the normal operation.

Even though most catastrophic faults lead to a complete cessation of droplet transportation, there exist differences between their corresponding erroneous behaviors. For instance, to test for the electrode-open fault, it is sufficient to move a test droplet from any adjacent cell to the faulty cell. The droplet will always be stuck during its motion due to the failure in charging the control electrode. On the other hand, if we move a test droplet across the faulty cells affected by an electrode-short fault, the test droplet may or may not be stuck depending on its flow direction. Therefore, to detect such faults, it is not enough to solve only the Hamiltonian path problem. In [Su 2005b], a solution based on Euler paths in graphs is described for detecting electrode shorts.

Despite its effectiveness for detecting electrode shorts, testing based on an Euler path suffers from long test application time. This approach uses only one droplet to traverse the microfluidic array, irrespectively of the array size. Fault diagnosis is carried out by using multiple test application steps and adaptive Euler paths. Such a diagnosis method is inefficient since defect-free cells are tested multiple times. Moreover, the test method leads to a test plan that is specific to a target biochip. If the array dimensions are changed, the test plan must be completely altered. In addition, to facilitate chip testing in the field, test plans need to be programmed into a microcontroller. However, the hardware implementations of test plans from [ $\mathrm{Su} 2005 \mathrm{a}]$ are expensive, especially for low cost, disposable biochips.

More recently, a cost-effective testing methodology referred to as "parallel scan-like test has been proposed [Xu 2007b]. The method is named thus because it manipulates multiple test droplets in parallel to traverse the target microfluidic array, just as test stimuli can be applied in parallel to the different scan chains in an integrated circuit.

Application to a Fabricated Biochip: The parallel scan-like test method has been applied to a fabricated biochip. The chip-under-test is a PCB microfluidic platform for DNA sequencing, as shown in Fig. 2. The platform consists of a $7 \times 7$ array, 8 reservoirs and routing electrodes that connect reservoirs to the array. A total of 9 cells are reserved for grounding, and they are not available for droplet transportation. 
Table 1. Examples of fault models for digital microfluidic lab-on-chip [Xu 2007a].

\begin{tabular}{|c|c|c|c|c|}
\hline $\begin{array}{l}\text { Cause of } \\
\text { defect }\end{array}$ & $\begin{array}{l}\text { Defect } \\
\text { type }\end{array}$ & $\begin{array}{l}\text { Number } \\
\text { of cells }\end{array}$ & $\begin{array}{l}\text { Fault } \\
\text { model }\end{array}$ & $\begin{array}{l}\text { Observable } \\
\text { error }\end{array}$ \\
\hline $\begin{array}{l}\text { Excessive actuation } \\
\text { voltage applied to an electrode }\end{array}$ & Dielectric breakdown & 1 & $\begin{array}{l}\text { Droplet-electrode short (a } \\
\text { short between the droplet } \\
\text { and the electrode) }\end{array}$ & $\begin{array}{l}\text { Droplet undergoes electrolysis, which } \\
\text { prevents its further transportation }\end{array}$ \\
\hline $\begin{array}{l}\text { Electrode actuation for } \\
\text { excessive duration }\end{array}$ & $\begin{array}{l}\text { Irreversible charge } \\
\text { concentration on an electrode }\end{array}$ & 1 & $\begin{array}{l}\text { Electrode-stuck-on (the } \\
\text { electrode remains constantly } \\
\text { activated) }\end{array}$ & $\begin{array}{l}\text { Unintentional droplet operations or } \\
\text { stuck droplets }\end{array}$ \\
\hline $\begin{array}{l}\text { Excessive mechanical force } \\
\text { applied to the chip }\end{array}$ & $\begin{array}{l}\text { Misalignment of parallel plates } \\
\text { (electrodes and ground plane) }\end{array}$ & 1 & $\begin{array}{l}\text { Pressure gradient (net static } \\
\text { pressure in some direction) }\end{array}$ & $\begin{array}{l}\text { Droplet transportation without } \\
\text { activation voltage }\end{array}$ \\
\hline Coating failure & Non-uniform dielectric layer & 1 & $\begin{array}{l}\text { Dielectric islands } \\
\text { (islands of Teflon coating) }\end{array}$ & $\begin{array}{l}\text { Fragmentation of droplets and their } \\
\text { motion is prevented }\end{array}$ \\
\hline \multirow{3}{*}{$\begin{array}{l}\text { Abnormal } \\
\text { metal layer } \\
\text { deposition } \\
\text { and etch } \\
\text { variation } \\
\text { during } \\
\text { fabrication }\end{array}$} & Grounding Failure & 1 & $\begin{array}{l}\text { Floating droplets (droplet are } \\
\text { not anchored ) }\end{array}$ & Failure of droplet transportation \\
\hline & Broken wire to control source & 1 & $\begin{array}{l}\text { Electrode open (electrode } \\
\text { actuation is not possible) }\end{array}$ & $\begin{array}{l}\text { Failure to activate the } \\
\text { electrode for droplet } \\
\text { transportation }\end{array}$ \\
\hline & $\begin{array}{l}\text { Metal connection between two } \\
\text { adjacent electrodes }\end{array}$ & 2 & $\begin{array}{l}\text { Electrode short (short } \\
\text { between electrodes) }\end{array}$ & \multirow{2}{*}{$\begin{array}{l}\text { A droplet resides in } \\
\text { the middle of the two shorted } \\
\text { electrodes, and its transport along one } \\
\text { or more directions } \\
\text { cannot be achieved }\end{array}$} \\
\hline $\begin{array}{l}\text { Particle contamination or } \\
\text { liquid residue }\end{array}$ & $\begin{array}{l}\text { A particle that connect two } \\
\text { adjacent electrodes }\end{array}$ & 2 & Electrode short & \\
\hline \multirow{2}{*}{$\begin{array}{l}\text { Protein adsorption during } \\
\text { bioassay [17] }\end{array}$} & \multirow{2}{*}{$\begin{array}{l}\text { Sample residue on electrode } \\
\text { surface }\end{array}$} & \multirow[t]{2}{*}{1} & Resistive open at electrode & Droplet transportation is impeded. \\
\hline & & & Contamination & $\begin{array}{l}\text { Assay results are outside the range of } \\
\text { possible outcomes }\end{array}$ \\
\hline
\end{tabular}

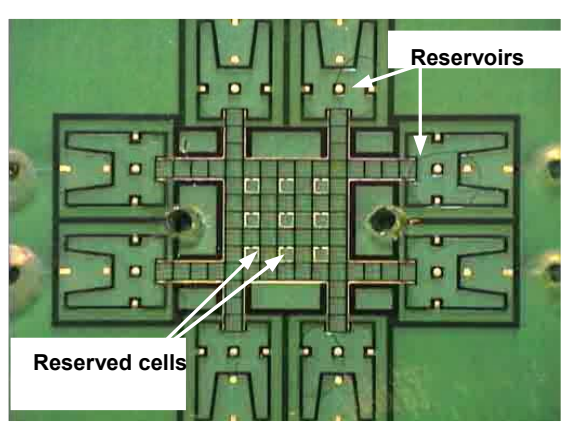

Fig. 2: Fabricated lab-on-chip for DNA sequencing used to demonstrate parallel scan-like testing.

As a baseline, Euler-path-based testing was applied to this chip [Xu 2007b]. The test procedure takes 57 seconds, assuming a (typical) $1 \mathrm{~Hz}$ electrode-actuation frequency. Next, parallel scan-like test was applied to this chip. The test application procedure takes 46 seconds, again for a $1 \mathrm{~Hz}$ actuation frequency.

Next we evaluate the time needed for fault diagnosis for the two methods. In [Xu 2007b], a fabricated chip was used, which was known a priori to contain one defect. For the Euler-path-based method, binary search was carried out to locate the defective cell. A total of seven iterations were needed and the total diagnosis time was $173 \mathrm{~s}$. On the other hand, parallel scan-like test can simply determine the defect site from test-outcome readouts. No additional diagnosis steps are needed and the diagnosis time is the same as the testing time, i.e., $44 \mathrm{~s}$, which correspond to a $75 \%$ reduction compared to [ $\mathrm{Su} 2005$ ].

A drawback of the above "structural" test methods is that they focus only on physical defects, and they overlook module functionality. Therefore, these methods can only guarantee that a biochip is defect-free. However, a defect-free microfluidic array can also malfunction in many ways. For example, a defect-free reservoir may result in large volume variations when droplets are dispensed from it. A splitter composed of three defect-free electrodes may split a big droplet into two droplets with significantly unbalanced volumes. These phenomena, referred to as malfunctions, are not the result of electrode defects. Instead, they are activated only for certain patterns of droplet movement or fluidic operations. Such malfunctions can have serious consequences on the integrity of bioassay results.

\section{Functional Testing}

Functional testing involves test procedures to check whether groups of cells can be used to perform certain operations, e.g., droplet mixing and splitting. For the test of a specific operation, the corresponding patterns of droplet movement are carried out on the target cluster of cells. If a target cell cluster fails the test, e.g., the mixing test, we label it as a malfunctioning cluster.

As in the case of structural testing, fault models must be developed for functional testing. Malfunctions in fluidic operations are identified and included in the list of faults; see Table 2.

Functional test methods to detect the defects and malfunctions have recently been developed. In particular, dispensing test, mixing test, splitting test, and capacitive sensing test have been described in $[\mathrm{Xu} 2007 \mathrm{a}]$ to address the corresponding malfunctions.

Some Experimental Results: Functional test methods were applied to a PCB microfluidic platform for the Polymerase Chain Reaction (PCR), as shown in Fig. 3. The platform consists of two columns and two rows of electrodes, three reservoirs, and routing electrodes that connect the reservoirs to the array. A dispensing malfunction is shown in Fig. 4.

An illustration of the mixing and splitting test is shown in Fig. 5. The bottom row was first targeted and five test droplets were dispensed to the odd electrodes, as shown in Fig. 5(a). Next, splitting test for the even electrodes was carried out. Droplets were split and merged on the even electrodes. In Fig. 5(b), we see a series of droplets of the same volume resting on the even electrodes, which means that all the odd electrodes passed the splitting test, and merging at the even electrodes worked well. However, when the splitting test was carried out on the even electrodes, a large variation in droplet volume was observed on the $3^{\text {rd }}$ and $5^{\text {th }}$ electrodes; see Fig. 5(c). This variation implied a malfunction, leading to unbalanced splitting on the $4^{\text {th }}$ electrode. The malfunction was detected when the droplets were routed to the capacitive sensing circuit. The $4^{\text {th }}$ electrode on the bottom row was marked as an unqualified splitting site. 
Table 2. Functional fault models [Xu 2007a].

\begin{tabular}{|c|c|c|c|c|}
\hline $\begin{array}{l}\text { Cause of } \\
\text { malfunction }\end{array}$ & $\begin{array}{l}\text { Malfunction } \\
\text { type }\end{array}$ & $\begin{array}{l}\text { Number } \\
\text { of cells }\end{array}$ & $\begin{array}{l}\text { Fault } \\
\text { model }\end{array}$ & $\begin{array}{l}\text { Observable } \\
\text { error }\end{array}$ \\
\hline $\begin{array}{l}\text { Electrode actuation for } \\
\text { excessive duration }\end{array}$ & $\begin{array}{l}\text { Irreversible charge } \\
\text { concentration on the dispensing } \\
\text { electrode }\end{array}$ & 3 & $\begin{array}{l}\text { Dispensing-stuck-on (droplet } \\
\text { is dispensed by not fully cut } \\
\text { off from the reservoir) }\end{array}$ & $\begin{array}{l}\text { No droplet can be dispensed from the } \\
\text { reservoir }\end{array}$ \\
\hline $\begin{array}{l}\text { Electrode shape variation in } \\
\text { fabrication }\end{array}$ & Deformity of electrodes & 3 & $\begin{array}{l}\text { No overlap between droplets } \\
\text { to be mixed and center } \\
\text { electrode }\end{array}$ & Mixing failure \\
\hline $\begin{array}{l}\text { Electrode electrostatic } \\
\text { property variation in } \\
\text { fabrication }\end{array}$ & Unequal actuation voltages & 3 & $\begin{array}{l}\text { Pressure gradient (net static } \\
\text { pressure in some direction) }\end{array}$ & Unbalanced volumes of split droplets \\
\hline Bad soldering & $\begin{array}{l}\text { Parasitic capacitance in the } \\
\text { capacitive sensing circuit }\end{array}$ & 1 & $\begin{array}{l}\text { Oversensitive or insensitive } \\
\text { capacitive sensing }\end{array}$ & False positive/negative in detection \\
\hline
\end{tabular}

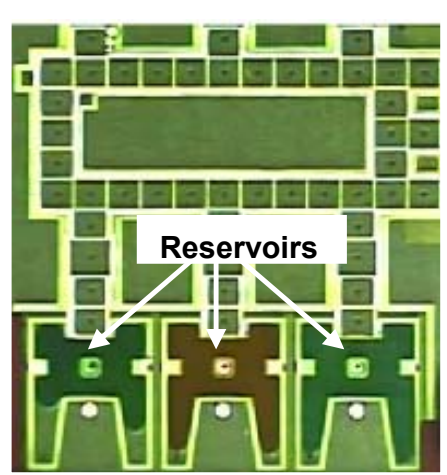

Fig. 3: Fabricated lab-on-chip used for PCR.

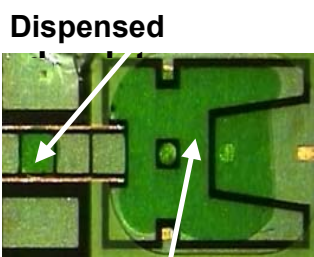

Reservoir

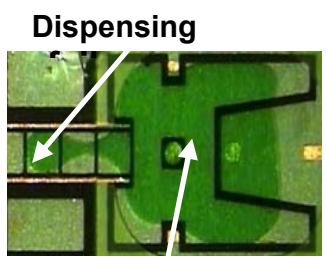

Reservoir
Fig. 4: Illustration of (a) normal dispensing and (b) dispensing failure, for a fabricated lab-on-chip.

\section{Conclusions}

We have presented a survey of research on fault tolerance in digital microfluidic lab-on-chip systems. Common defects have been identified and related to logical fault models. Based on these fault models, test techniques for emerging lab-on-chip devices and digital microfluidic modules have been presented. The use of these test techniques for fabricated devices has been highlighted.

\section{Acknowledgments}

The author thanks a number of colleagues at Duke University and at Advanced Liquid Logic, Inc., who have contributed to this work. They include Dr. Fei Su, Tao Xu, Yang Zhao, William Hwang, Dr. Phil Paik, Dr. Vamsee Pamula, and Prof. Richard Fair. This work was supported in part by the National Science Foundation under grants IIS-0312352 and CCF-0541055.

\section{References}

[Fair 2003] R. B. Fair et al., "Electrowetting-based on-chip sample for Digital Microfluidic Circuits", Journal of MEMS, vol. 12, Feb. 2003, pp. 70-80.

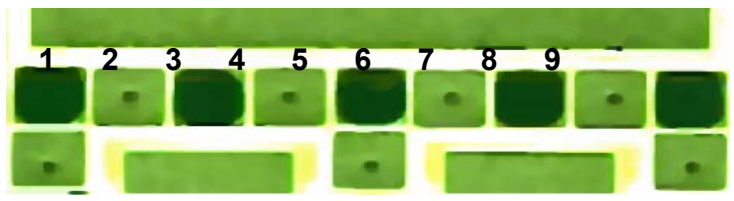

(a)

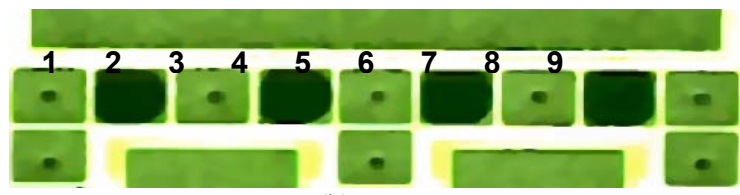

(b)

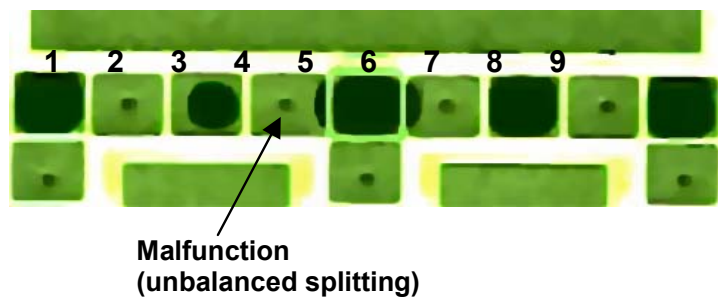

(c)

Fig. 5: Mixing and splitting test for a fabricated PCR chip.

[Verpoorte 2003] E. Verpoorte and N. F. De Rooij, "Microfluidics meets MEMS”, Proc. IEEE. vol. 91, pp. 930-953, 2003.

[Dittrich 2006] P. S. Dittrich, K. Tachikawa, and A. Manz, "Micro total analysis systems: latest advancements and trends", Analytical Chemistry, vol. 78, pp. 3887-3908, 2006.

[Cho 2003] S.K. Cho, H. Moon, and C.J. Kim. Creating, transporting, cutting, and merging liquid droplets by electrowetting-based actuation for digital microfluidic circuits", Journal of MEMS, vol. 12, pp. 70-80, 2003. [Kerkhoff 2007] H. G. Kerkhoff, "Testing of microelectronic-biofluidic systems", IEEE Design \& Test of Computers, vol. 24, pp. 72-82, 2007.

[Su 2006] F. Su et al., "Concurrent testing of digital microfluidics-based biochips", ACM Trans. Design Automation of Electronic Systems, vol 11, pp. 442-464, 2006.

[Su 2005a] F. Su, S. Ozev and K. Chakrabarty, "Ensuring the operational health of droplet-based microelectrofluidic biosensor systems", IEEE Sensors, 2005, vol. 5, pp. 763-773.

[Su 2005b] F. Su et al., "Built-in self-test and defect tolerance in molecular electronics-based nanofabrics", Journal of Electronic Testing: Theory and Applications, vol. 23, pp. 145-161, 2007.

[Xu 2007a] T. Xu and K. Chakrabarty, "Functional testing of digital microfluidic biochips", Proc. IEEE International Test Conference, 2007.

[Xu 2007b] T. Xu and K. Chakrabarty, "Parallel scan-like test and multiple-defect diagnosis for digital microfluidic biochips", IEEE Trans. Biomedical Circuits and Systems, vol. 1, pp. 148-158, June 2007. 